\title{
Impact of COVID-19 pandemic on Radiation Oncology Residency- Experience and Perspective from a Tertiary Cancer Centre of Northeast India
}

\author{
Biswajit Sarma, Hima Bora, Dhiru Talukdar, Luri Borah, Moumita Paul, \\ Prashasti Sharma, Jyotiman Nath
}

Dr. B Boroaah Cancer Institute, Guwahati, India.

\begin{abstract}
The novel corona virus disease 2019 has emerged as a global pandemic affecting the healthcare system. It became extremely difficult to carryout comprehensive cancer care services in the midst of this pandemic. As resident trainees in the department of Radiation Oncology, we faced many difficulties during this unprecedented crisis. We the radiation oncology residents from a tertiary cancer centre of northeast India want to highlight our preparedness and precautionary measures taken to ensure uninterrupted patient services and the impact of this crisis on our academics.
\end{abstract}

Keywords: COVID-19- radiation oncology- resident- cancer

\section{Introduction}

The novel Corona virus disease 2019 (COVID-19) that originated in the Wuhan, Hubei Province of China is emerging as a global public health problem. It is caused by the severe acute respiratory syndrome coronavirus 2 (SARS-CoV-2) [1]. The family of Coronaviridae consists of a group of large, single, plus-stranded RNA-viruses which have been isolated from several species; common symptoms in humans include common cold, fever and diarrheal illness. The first reported case of COVID - 19 from China came in late December 2019 and subsequently it spread globally reporting cases from European region during late January 2020. In India the first case of corona virus disease was detected in Kerala on $30^{\text {th }}$ January 2020. The World Health Organization (WHO) subsequently declared the COVID-19 outbreak a pandemic on 11 March 2020 [2]. As of $5^{\text {th }}$ July, 2020 in India reported total $6,73,932$ confirmed cases of COVID-19 with COVID related death of 19,279 cases [3]. The state of Assam has also reported 11,002 confirmed cases till $5^{\text {th }}$ of July 2020 [4]. To break the chain of transmission of the disease the Govt. of India declared total nationwide lockdown on March $24^{\text {th }} 2020$ in a phased manner.
Dr Bhubaneswar Borooah Cancer Institute is a tertiary cancer center in state of Assam. The institute caters high volume of patients from all the northeastern states as well as from a part of West Bengal.

Indian Council of Medical Research (ICMR) has released guidelines for health care institutions across the nation on strategies to cope with COVID-19 and minimize its risk to patients and health care workers [5]. People with cancer have a higher susceptibility to get infected with COVID-19, and their outcomes are relatively poor [6]. Due to this unprecedented crisis, the residents in radiation oncology department faced challenges to manage cancer patients.

\section{Approach Towards Patient Care}

At our institute COVID-19 having a tremendous impact on the patients including possible postponement of oncologic surgery, as well as transfer of all possible inpatient oncology services to makeshift outpatient facilities. During this pandemic the department formulated some policies adhering to available literature and published ICMR and Govt. guidelines for smooth functioning. As an

\footnotetext{
Corresponding Author:

Dr. Prashasti Sharma

Dr. B Boroaah Cancer Institute, Guwahati, India.

Email: prashasti1993@yahoo.com
} 
integral part of the patient care the service of the resident doctors continued without fail following strict hygiene and safety precautions.

The Govt. of India formulated standard operating protocol (SOP) for reallocation of residents/ post graduate students as part of hospital management of COVID [7]. As per this protocol, we the radiation oncology residents fall under category $\mathrm{C}$ and accordingly we have been posted in a COVID screening area on shift basis. In the screening area we used to take history of fever and recent travel of all the patients and attendants. Temperature screening was done by infrared thermometer. Anyone with body temperature over $37.3{ }^{\circ} \mathrm{C}$ and recent travel history were transferred to a newly established fever clinic or isolation ward. Then persons with suspected COVID infections were referred to Gauhati Medical College and Hospital for further investigation and management. In the first week of July 2020, the government of Assam through National Health Mission (NHM) has provided two TrueNAT machine with necessary consumables in our institute for COVID - 19 testing. Accordingly the chairmen Atomic Energy Commission (AEC) and secretary Department of Atomic Energy, Govt. of India has accorded approval for COVID-19 testing laboratory in our institute. This development will increase the preparedness of the institute in early testing, tracing of COVID-19 positive patient.

A resident in radiation oncology department have to work diversely in various places like outpatient department, simulation room, treatment delivery room etc. Most of the places are potential areas of transmission of the disease. Sudden deployment of social distancing norms leads to panic and anxiety among different residents during initial days of this pandemic. However with increasing knowledge and training of different preventive measures gradually helped us to gain confidence in patient management.

To reduce the risk of disease transmission among the health care workers and the patients the hospital authority advised strict protocols at different facilities inside the hospital. Social distancing was maintained at Radiotherapy OPD and waiting areas. Only one patient is examined at one time with only one attendant. No patient or attendant is allowed inside the consultation room without facemask. We have been provided with N-95 mask and face shield for consultation.

The radiotherapy machine and the waiting area are potential area of human crowd. Those patients taking radiotherapy treatment in the regular basis are educated about the need of social distancing, hand hygiene. While verifying and delivering treatment in the machine room, we the residents as well as the technologists take extra cautions especially in high risk patients like tracheostomized ones. All radiotherapy technologist and support staff were mobilized and trained in infection prevention and control practices.

The faculties of our department formulated few institutional protocols based on availability of evidence that helped us in reducing workload and total treatment time without compromising disease outcome. As a part of this we started using hypofractionated radiotherapy regimens for sites like cancer breast and prostate [8-9]. As the need of the hour we the residents arranged group discussions and online meetings to understand these guidelines with the help of published literature.

\section{Impact on Academics and Training}

Residency training demands balance between delivering patient care and continuous learning activities. Academic activities include bedside clinics, group discussions, seminars, journal clubs etc. Multidisciplinary tumour board is an integral learning platform for oncology residents. Because of the current social distancing measures various clinical activities like case presentations, tumour board meetings, seminars were cancelled. To overcome these deficiencies, the faculties have arranged few academic sessions via online platform. We residents took part in different webinars, online academic activities using different applications available in electronic gadgets to get up-to-date with recent advances. Our institute being a member of national cancer grid (NCG) encouraged all the residents to attend the NCG webinars to get updated with COVID preparedness as well as oncologic advances. However, the residents are facing difficulty in accepting this new format of e-learning.

With the postponement of university examination of final year residents, the stresses among them are increasing with the dilemma and uncertainity about their future plans.

In conclusion, the COVID-19 pandemic has severely impacted the healthcare system worldwide. It became extremely difficult to carryout comprehensive cancer care services specially the radiation therapy procedures as these are usually long and resource intensive. The residents of the radiation oncology department are also facing challenges to carry out a balance between patient care and learning. It is important for the trainees to remain connected with peers and friends via all possible medium so that social distancing doesn't results in social isolation. Time out of the daily work should be utilized for nurturing personal and family relationships, light physical exercise and recreational activities to help to maintain peace of body and soul.

\section{References}

1. Andersen KG, Rambaut A, Lipkin WI, Holmes EC, Garry RF. The proximal origin of SARS-CoV-2. Nature Medicine. 202003 17;26(4):450-452. https://doi.org/10.1038/s41591020-0820-9

2. Darafsheh A, Lavvafi H, Taleei R, Khan R. Mitigating disruptions, and scalability of radiation oncology physics work during the COVID-19 pandemic. Journal of Applied Clinical Medical Physics. 2020 05 20;21(7):187-195. https:// doi.org/10.1002/acm2.12896

3. Coronavirus in India: Latest Map and Case Count [Internet]. Covid19india.org. 2020 [cited 7 July 2020]. Available from: https://www.COVID19india.org/.

4. [Internet]. 2020 [cited 7 July 2020]. Available from: https:// covid19.assam.gov.in/.

5. Indian Council of Medical Research, New Delhi [Internet]. Icmr.gov.in. 2020 [cited 7 July 2020]. Available from: https:// www.icmr.gov.in/. 
6. Liang W, Guan W, Chen R, Wang W, Li J, Xu K, Li C, Ai Q, Lu W, Liang H, Li S, He J. Cancer patients in SARSCoV-2 infection: a nationwide analysis in China. The Lancet Oncology. 2020 03;21(3):335-337. https://doi.org/10.1016/ s1470-2045(20)30096-6

7. [Internet]. Mohfw.gov.in. 2020 [cited 7 July 2020]. Available from: https://www.mohfw.gov.in/pdf/ COVID19SOPfordoctorsandnurses.pdf..

8. The UK Standardisation of Breast Radiotherapy (START) Trial B of radiotherapy hypofractionation for treatment of early breast cancer: a randomised trial. The Lancet. 2008 03;371(9618):1098-1107. https://doi.org/10.1016/s01406736(08)60348-7

9. Morgan SC, Hoffman K, Loblaw DA, Buyyounouski MK, Patton C, Barocas D, Bentzen S, Chang M, Efstathiou J, Greany P, Halvorsen P, Koontz BF, Lawton C, Leyrer CM, Lin D, Ray M, Sandler H. Hypofractionated Radiation Therapy for Localized Prostate Cancer: An ASTRO, ASCO, and AUA Evidence-Based Guideline. Journal of Clinical Oncology. 2018 Dec 01;36(34):3411-3430. https://doi. org/10.1200/jco.18.01097

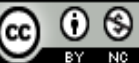

This work is licensed under a Creative Commons AttributionNon Commercial 4.0 International License. 